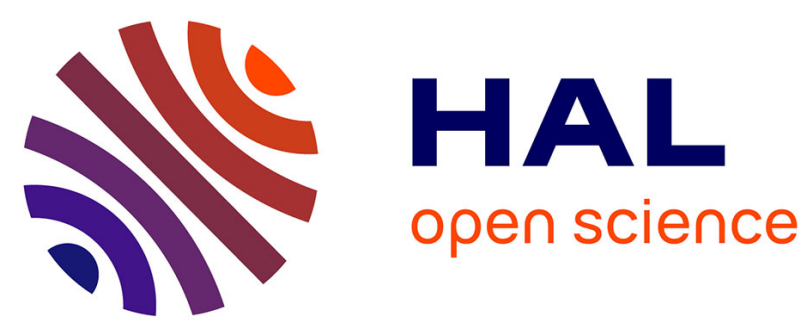

\title{
Near-UV Resonant Two-Photon Ionization Spectroscopy of Gas Phase Guanine: Evidence for the Observation of Three Rare Tautomers
}

\author{
M. Mons, F. Piuzzi, I. Dimicoli, L. Gorb, J. Leszczynski
}

\section{- To cite this version:}

M. Mons, F. Piuzzi, I. Dimicoli, L. Gorb, J. Leszczynski. Near-UV Resonant Two-Photon Ionization Spectroscopy of Gas Phase Guanine: Evidence for the Observation of Three Rare Tautomers. Journal of Physical Chemistry A, 2006, 110, pp.10921-10924. 10.1021/jp063738x . hal-00085677

\section{HAL Id: hal-00085677 https://hal.science/hal-00085677}

Submitted on 23 Jan 2007

HAL is a multi-disciplinary open access archive for the deposit and dissemination of scientific research documents, whether they are published or not. The documents may come from teaching and research institutions in France or abroad, or from public or private research centers.
L'archive ouverte pluridisciplinaire $\mathbf{H A L}$, est destinée au dépôt et à la diffusion de documents scientifiques de niveau recherche, publiés ou non, émanant des établissements d'enseignement et de recherche français ou étrangers, des laboratoires publics ou privés. 


\title{
Near-UV Resonant Two-Photon Ionization Spectroscopy of Gas Phase Guanine: Evidence for the Observation of Three Rare Tautomers
}

\author{
Michel Mons $^{\mathrm{a} *}$, François Piuzzi ${ }^{\mathrm{a}}$, Iliana Dimicoli, ${ }^{\mathrm{a}}$ Leonid Gorb $^{\mathrm{b}, \mathrm{c}, \mathrm{d}}$ and Jerzy Leszczynski ${ }^{\mathrm{b}}$ \\ ${ }^{a}$ Laboratoire Francis Perrin (URA CEA CNRS 2453), Service des Photons, Atomes et Molécules, CEA \\ Saclay, Bât. 522, 91191 Gif-sur-Yvette Cedex, France \\ ${ }^{b}$ Computational Centre for Molecular Structure and Interactions, Dept. of Chemistry, ${ }^{b}$ Jackson State \\ University, P.O. Box 17910, 1325 Lynch St., Jackson, MS 39217,USA \\ ${ }^{c}$ Ukrainian-American Laboratory of Computational Chemistry, Kharkiv, Ukraine, and Jackson, \\ Mississippi \\ ${ }^{d}$ Department of Molecular Biophysics, Institute of Molecular Biology and Genetics, National Academy \\ of Science of Ukraine, 150 Zabolotnogo Street, Kyiv 03143, Ukraine
}

\begin{abstract}
In the light of a recently published study on the IR spectroscopy of guanine in He droplets (Choi and Miller, JACS 2006, 128, 7320), the present letter proposes a new interpretation of the resonant two-photon ionization (R2PI) experiments on gas phase guanine, which is supported by quantum chemistry calculations. Whereas He droplet experiments detect the most stable forms, only one of these forms is observed (very marginally) in the R2PI spectrum, which is actually dominated by three less stable "rare" tautomers, whose stabilities lie in the 3-7 $\mathrm{kcal} / \mathrm{mol}$ range. The absence of the most stable forms in the R2PI spectrum suggests that a tautomer-dependent ultra-fast relaxation process takes place in the excited state of these stable tautomers. The present reinterpretation modifies qualitatively the picture of the excited state of guanine tautomers and should contribute to understand the deactivation mechanisms taking place in the excited state of DNA bases.
\end{abstract}

Understanding the near-UV absorption of DNA bases is a key issue for the study of photoinduced damages to DNA material. In this context, the near-UV spectrum of guanine in a supersonic expansion has been widely investigated using nanosecond laser resonant two-photon ionization (R2PI). ${ }^{1-8}$ Using the IR/UV double resonance technique, Nir et al. found vibrationally resolved contributions of three different tautomers (A, B and $\mathrm{C}$ in Fig. 1) and assigned them to 3 forms among the most stable of guanine : one hydroxy-amino (enol) form (either 3 or 4 ), and two oxoamino (keto) forms 2 and $\mathbf{1}$ respectively, by comparison between IR experimental and calculated spectra. ${ }^{3}$ Using the same experimental technique, our group confirmed the observation of the three forms $\mathrm{A}-\mathrm{C}$ and identified the contribution of a fourth tautomer D. ${ }^{6,8}$ Based on a comparison with the UV spectra of 7- and 9-methylated species $^{6}$ and of hydrates, ${ }^{8}$ we concluded to different assignments in terms of $7 / 9 \mathrm{H}$ tautomerism for the $\mathrm{A}, \mathrm{B}$ and $\mathrm{C}$ features: i.e., 5, 1 and 2, respectively. In particular, the red-most intense band system (A), assigned to the N9H enol form (either $\mathbf{3}$ or $\mathbf{4}$ ) by the de Vries' group was assigned by us to a higher energy form: $7 \mathrm{H}$ enol syn $\mathbf{5}$. Finally, the fourth tautomer D observed was assigned to the $9 \mathrm{H}$ enol form 4. ${ }^{6,8}$ Paradoxically, the R2PI spectrum of 9methylguanine $(9 \mathrm{MG})$ is a much simpler, narrow-range, band system (Fig. 1) and found to be due to a unique form: the same $9 \mathrm{H}$ enol anti $\mathbf{4}$ tautomer as the D form of

Chart 1. Most stable ${ }^{9-11}$ tautomers of guanine ${ }^{a}$
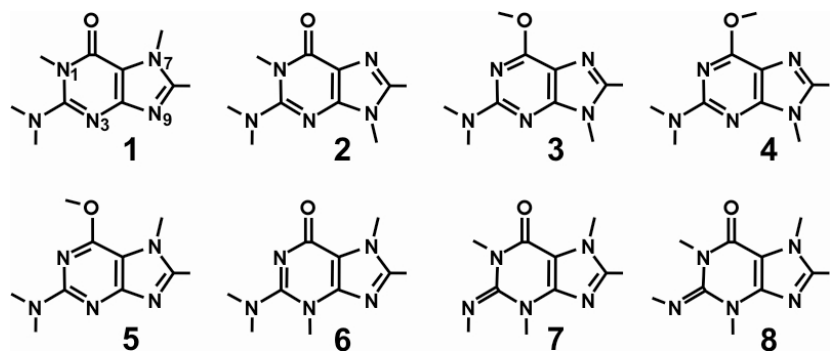

a: 1,2 and 6 are oxo-amino (keto); 3-5: hydroxy-amino (enol) and 7-8: oxo-imino forms. Relative energies ${ }^{9-11}$ are in the following energy ranges: 1$4: 0-1 \mathrm{kcal} / \mathrm{mol}, 5 \sim 3 \mathrm{kcal} / \mathrm{mol}$ and $\mathbf{6 - 8} \sim 7 \mathrm{kcal} / \mathrm{mol}$. The next forms are found to be in the $12 \mathrm{kcal} / \mathrm{mol}$ range. ${ }^{9}$ Although the precise relative energies depend on the level of theory used, these data provide an overview of the conformational landscape.

guanine. $^{6,8}$ The enol syn counterpart $\mathbf{3}$ and the biologically relevant keto form 2, calculated to be of similar stability, are surprisingly missing, which suggested that 9methylation could have a dramatic effect on the excited dynamics of these tautomers. ${ }^{8}$ However, the several theoretical studies focused on this point failed to account for this so-called 9-methylation effect. ${ }^{12,13}$ The issue was left at this point, until a very recent work by Roger Miller's group $^{14}$ provided relevant enlightening data, which enables 


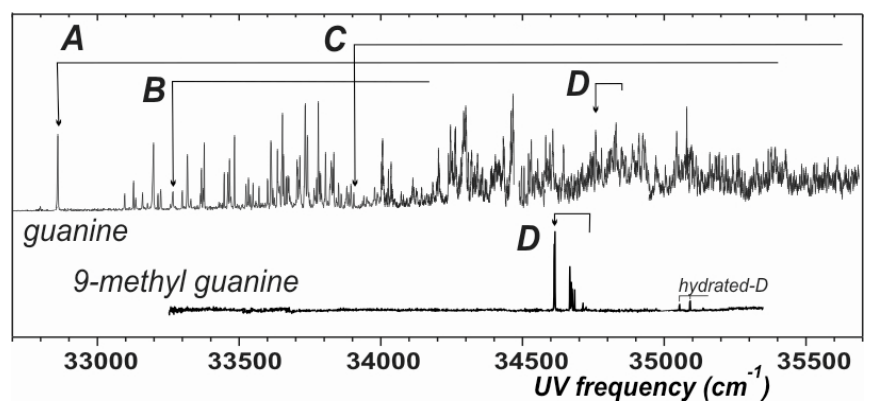

Figure 1. R2PI spectrum of guanine and 9-methylguanine as obtained with a ns-UV laser. ${ }^{6,8}$ The origins of the four tautomers identified are indicated by arrows. Assignments of A and D to forms $\mathbf{5}$ and $\mathbf{4}$, respectively, are based upon comparison with 7- and 9-methylated species. B and $\mathrm{C}$ features are assigned to oxo-imino conformers, $\mathbf{8}$ and $\mathbf{7}$ respectively, by comparison with theoretical calculations, see Fig. 2.

us to propose an elegant way to rationalize the puzzling UV R2PI spectroscopy of guanine.

R. Miller and his coworkers, working with molecules embedded in He droplets, have developed a very powerful method to assign conformational contributions in vibrational spectra, based upon comparison between the vibrational transition moment angles determined from experiment and theory. ${ }^{15}$ Applied to adenine and cytosine, these studies led to IR spectra nearly identical to those obtained in the gas phase, ${ }^{16}$ as expected from a soft matrix like suprafluid He. For guanine, the same methodology ${ }^{14}$ provides an unambiguous evidence for population of four tautomers under the $\mathrm{He}$ droplet environment. The very convincing agreement of the complete experimental set of data with the quantum chemistry calculations enables Choi and Miller to assign the IR spectrum (NH stretch region) to a mixture of the four most stable forms (1-4), namely the 7Hketo, 9Hketo, 9Henol syn and 9Henol anti tautomers (Fig. 2). ${ }^{14}$ The comparison with IR/UV double resonance spectroscopy, $3,4,6,8$ however, is quite intriguing. In contrast to what is generally observed for other molecules, the IR He droplet data are not found to match the IR/UV spectra (Fig. 2). Only one form observed in He droplets ${ }^{14}$ is also found in the R2PI experiment under isolated conditions: $:^{6,8}$ the $9 \mathrm{H}$ enol anti 4 tautomer observed in He droplets exhibits IR spectral features nearly identical to those of the D form observed in R2PI experiments, with relative frequency differences of the order of $1 \mathrm{~cm}^{-1}$ (Table 1). No such close counterpart can be found among the IR/UV spectra for the three other conformers (1-3) observed in He droplets (Fig. 2 ). Even the A form, assigned to an oxo-amino (enol) form, ${ }^{3,6}$ does not match the second oxo-amino rotamer 3 in He droplets. This demonstrates clearly that the A-C forms observed in the R2PI experiment are not the same tautomers as those observed in He droplets: in other words, the two experiments actually do detect different tautomer populations.

Since the R2PI spectra reported are all mass-selected, the influence of impurities or of fragmenting clusters, whose UV signature would be seen on both cluster and guanine ion channels is easily ruled out. Therefore, the immediate consequence is that the forms (A-C) which are responsible for the intense features observed in the R2PI spectrum of guanine should actually be due to high-energy "rare" tautomers, whose weak population cannot be detected under cold $\mathrm{He}$ droplet conditions. In the following, we will propose an assignment of $\mathrm{A}-\mathrm{C}$ in terms of tautomers and discuss the possible reasons for the absence of the most stable tautomers in the R2PI spectrum.

Assignment of the A-D forms observed in the R2PI spectra. Our assignment of the D species to the $9 \mathrm{Henol}$ anti form $4^{6,8}$ by comparison with the UV spectrum of $9 \mathrm{MG}$ is in agreement with the independent analysis of Choi and Miller. ${ }^{14}$ This form is the only form observed simultaneously in both experiments. Concerning A, again the comparison with $7 \mathrm{MG}$ led us to assign it to the $7 \mathrm{H}$ enol syn form 5. ${ }^{6}$ The low stability argument of this form, which could be opposed so far, now obviously disappears, since, apart from $\mathrm{D}$, the observed tautomers must be rare forms. With respect to $\mathrm{B}$ and $\mathrm{C}$ features, the assignment in terms of oxo tautomerism is not questionable because of the absence of the remarkable features in the $3580 \mathrm{~cm}^{-1}$ region, characteristic of the hydroxy forms (Fig. 2). , $^{3,6,8}$ Again, comparison with the IR spectra of tautomers $\mathbf{1}$ and 2, measured by Choi and Miller, is informative, in particular concerning the symmetric and asymmetric $\mathrm{NH}_{2}$ stretch bands (Fig. 2). This $\mathrm{NH}_{2}$ doublet, easily detected by Choi and Miller, ${ }^{14}$ is not present in the IR/UV spectra of forms $B$ and $C .{ }^{3,6}$ This suggests that the rare tautomers responsible for these features might be oxo-imino species, like $\mathbf{7}$ or $\mathbf{8}$, rather than oxo-amino forms, as considered so far. . $^{3,4,6,8}$

In order to confirm this point and to propose a more refined assignment, quantum chemistry calculations of the optimized geometries and IR harmonic frequencies of the 18 forms have been carried out at the B3LYP/6-31+G(d) level of theory (Table S1), which was previously successfully used to account for the enol rotamers (3-5) of guanine derivatives. ${ }^{8}$ The results, depicted to the relative weakness of the imino $\mathrm{NH}$ bond, was not observed. ${ }^{4,6}$ The $\mathrm{N} 3 \mathrm{H}$ band, however, gives rise to a characteristic prominent absorption in the $3490 \mathrm{~cm}^{-1}$ range. In spite of a strong similarity between the two IR spectra, the details of the band patterns ${ }^{3}$ are well reproduced by theory, suggesting to assign the B and C band systems of Fig. 1 to the 8 and 7 forms respectively. One will notice that the other rare $7 \mathrm{H}$ oxo-amino form 6 does not match any IR spectrum experimentally available and is therefore observed neither in He droplets, nor in R2PI spectra.

The impressive match of the theoretical IR spectra with their experimental counterparts provides a good confidence for the assignment and illustrates the level of prediction which can be expected from quantum chemistry. The poor agreement observed previously, together with spectroscopic inconsistencies discussed in Ref ${ }^{6}$, were actually the indication of a misassignment. These clues were discarded on the legitimate basis that species observed under supersonic expansion conditions should be a priori the most stable species, namely forms $\mathbf{1}$ and $\mathbf{2}$.

The present reassignment also solves the puzzling socalled 9-methylation effect ${ }^{8}$ : the most intense band systems in R2PI spectra do not correspond to the most stable forms but to rare $7 \mathrm{H}$ tautomers: the biologically relevant $9 \mathrm{H}$ keto 2 and the 9Henol syn $\mathbf{3}$ forms are therefore missing in both the R2PI spectrum of 9-methylguanine and that of guanine.

Gas phase R2PI vs. He droplets. There is no obvious reason to expect that the tautomer populations should be so different in the two experiments and the explanation for the dichotomy observed should be found elsewhere. The detection schemes in both experiments are quite different. 


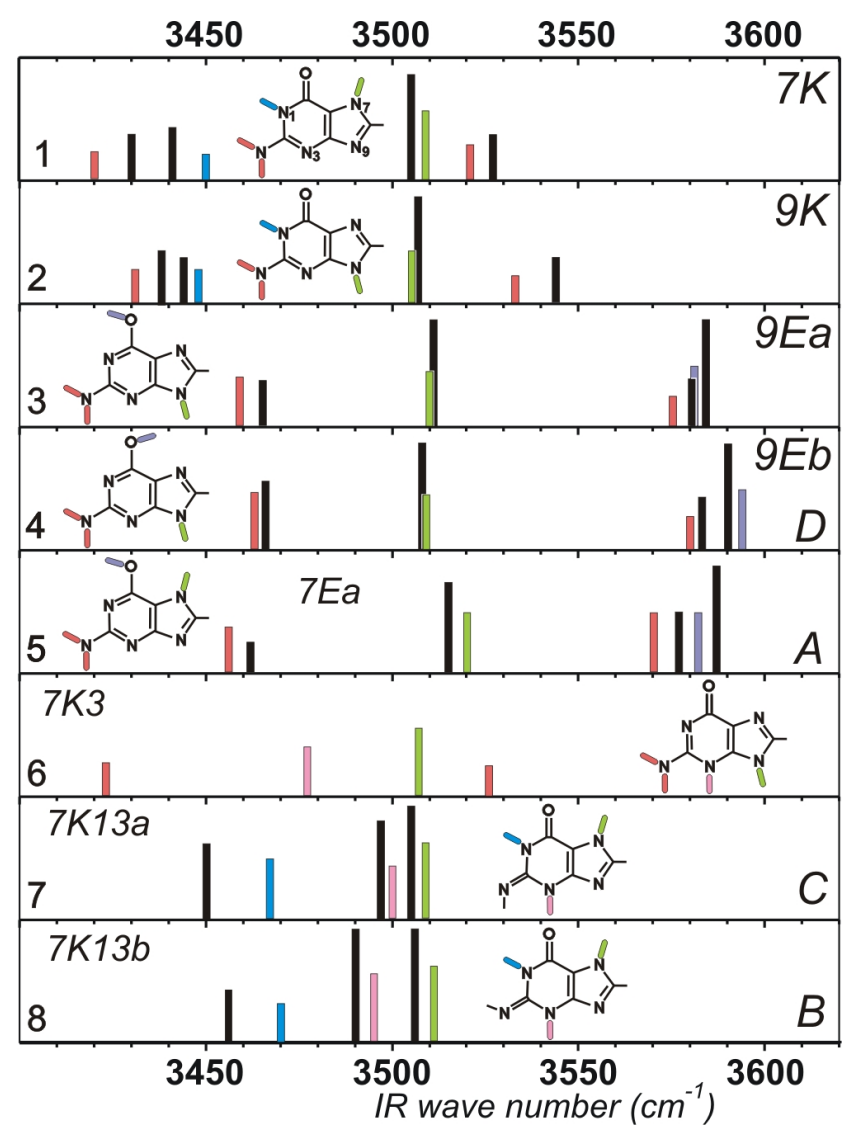

Figure 2. Theoretical IR spectra (B3LYP/6-31+G(d) harmonic frequencies of the $\mathrm{NH} / \mathrm{OH}$ stretches) of the 8 most stable forms of Chart 1 (color) compared to the spectra of the seven tautomers of guanine observed experimentally (black). The top four panels correspond to the 4 tautomers observed by Choi and Miller in He droplets (order of decreasing stability). ${ }^{14}$ Panels 5, 7 and 8 match the experimental IR data of forms A, C and B, respectively, observed in the R2PI spectrum of guanine. ${ }^{3,6,8}$ The D form observed in R2PI corresponds to the G9Eb form observed by Choi and Miller and exhibits the same band positions at the scale of the figure (the weak band at $3466 \mathrm{~cm}^{-1}$ could not be observed in our modest signal-to-noise ratio IR/UV spectrum ${ }^{6}$ ). Colors indicate the nature of the stretching modes: $\mathrm{NH}_{2}$ : red; N7/9H: green; N1H: blue, OH: violet, N3H: pink. Labeling of the spectral features on the right are those of Choi and $\mathrm{Miller}^{14}$ (He droplet data) as well as those of Mons et al. ${ }^{6}$ (R2PI data). Calculated NH and $\mathrm{OH}$ stretch frequencies are scaled by adjusted specific factors (0.963 and 0.971 respectively). Calculated spectra are displayed on an arbitrary smaller intensity scale for the sake of clarity.

In He droplet studies, IR absorption spectra are measured as a directly consequence of the energy absorbed. In contrast, gas phase IR/UV double resonance measures population depletions, which are monitored using a selective detection process based on UV absorption, namely by collecting either fluorescence from the excited state or ions produced through a R2PI scheme. This procedure implicitly requires that the lifetime of the excited state is long enough for allowing fluorescence emission or photoionisation during the laser pulse. In the R2PI case, the ionization potential (IP) of the species probed should also not be too high to be reached by two-photon excitation.

The absence of the most stable forms (1-3) in the R2PI experiments can be due a priori to several factors: i) a high IP for these species could forbid the two-photon ionization, ii) unfavorable Franck-Condon overlap might forbid the adiabatic excitation to the $\pi \pi^{*}$ state and therefore lead to a significantly blue-shifted, congested and complicated spectrum iii) additionally, an intrinsic ultrafast relaxation process $^{17}$ can quench the excited state population either as soon as the origin of the $\pi \pi^{*}$ state or after some excess energy and make therefore the absorption spectrum either severely broadened or quite narrow-ranged, and, finally, iiii) the absorption features may not be located in the spectral region probed so far. All these factors might combine together to forbid us the observation of the stable tautomers in the previous experiments, even if, so far, a wide spectral range has been investigated. Using R2PI, the 32150-35700 $\mathrm{cm}^{-1}$ range has been scanned, including a two-color R2PI scheme, ${ }^{1}$ in order to keep the capability to photoionize high IP species (second color at $193 \mathrm{~nm}$ ). The upper part of this range $\left(32700\right.$ to $35700 \mathrm{~cm}^{-1}$ ) region was also probed by fluorescence, which is not impeded by the high IP issue. A possible explanation for the missing features might be that the keto forms $\mathbf{1}$ and $\mathbf{2}$ exhibit very red-shifted UV transitions, in a region not yet investigated, even if this does not seem to be a definitive argument for three reasons. First, the $\pi \pi^{*}$ adiabatic transitions of the 1-4 conformers have been predicted to lie in the same energy range. ${ }^{12,18}$ Second, calculations also suggest a very large change in equilibrium geometry between the ground and the first $\pi \pi^{*}$ state. ${ }^{11-13,17-}$ ${ }^{21}$ Consequently, the Franck-Condon envelop is expected to be broad, comparable in spread to that observed by R2PI for the A-C forms. ${ }^{1,3-8}$ Finally, the second rotamer of the $9 \mathrm{H}$ enol-amino 3 form, whose spectrum is expected to lie in the vicinity of its twin form 4 , could not be detected, neither in guanine nor in $9 \mathrm{MG}$.

All these facts suggest the occurrence of a fast nonradiative relaxation of the excited state of the 1-3 forms which would prevent their observation in both the R2PI and fluorescence experiment. This is supported by the following points: First, the weird narrow range spectrum of the D form (4), composed of a few fluorescent bands in guanine and $9 \mathrm{MG}$, compared to the rich and long spectra of forms A or C (Fig. 1), suggests a dramatically sudden onset of relaxation occurring a few hundreds of wavenumbers above the $\pi \pi^{*}$ origin transition. Recent calculations indicate that in the biologically relevant $\mathbf{2}$ form, an internal conversion process, controlled by a conical intersection $(\mathrm{CI})$ with the ground state, is expected to occur close to the origin of the excited state $(\sim 1.7 \mathrm{kcal} / \mathrm{mol}) .{ }^{17}$ Such a process would qualitatively account for the sudden quenching of the excited state of D and also possibly for the 3 other forms since one can expect the location of the conical intersection to be tautomer-dependent. A CI located closer to the origin would make the excited state ultrashort-lived, forbidding its observation by R2PI or fluorescence. This is also consistent with the ultrashort lifetime measured for the $\pi \pi^{*}$ excited state of guanine (actually a mixture of its four most stable tautomers) at $267 \mathrm{~nm}$, which is the shortest of all the DNA bases. $^{22}$

In summary, the present letter rationalizes the gas phase and $\mathrm{He}$ droplet measurements of the literature on guanine in terms of observation of seven tautomers. Whereas $\mathrm{He}$ droplet experiments detect the most stable (1-4) tautomers, only one of these forms (4) is observed (very marginally) in the R2PI spectrum, which is actually dominated by three less stable "rare" tautomers $(\mathbf{5}, \mathbf{7}$ and $\mathbf{8})$, whose stabilities lie in a $3-7 \mathrm{kcal} / \mathrm{mol}$ range. The present finding radically modifies the qualitative picture of the excited state dynamics of the guanine tautomers suggesting the 
Table 1. Experimental IR band frequencies vs. theoretical harmonic NH stretch frequencies (B3LYP/6-31+G(d) level) for the four tautomers observed in the R2PI experiments. ${ }^{3,6}$

\begin{tabular}{|c|c|c|c|c|c|}
\hline $\begin{array}{l}\text { Tautomer / } \\
\text { UV band system } \\
\text { origin transition }\end{array}$ & $\begin{array}{l}\text { Experimental } \\
\text { Frequency } \\
\text { He droplet }\end{array}$ & $\begin{array}{l}\text { Experimental } \\
\text { Frequency } \\
\text { R2PI }\end{array}$ & $\begin{array}{l}\text { Scaled } \\
\text { Harmonic } \\
\text { frequency } \\
\left(\mathrm{cm}^{-1}\right)^{\mathrm{a}}\end{array}$ & $\begin{array}{l}\text { Intensity } \\
(\mathrm{km} / \mathrm{mol})\end{array}$ & Assignment \\
\hline (9H enol anti) & 3583.2 & 3583 & 3580 & 45 & $\mathrm{NH}_{2}$ antisym. \\
\hline D & 3509.6 & 3508 & 3509 & 73 & N9H \\
\hline origin : $34755 \mathrm{~cm}^{-1}$ & 3466.1 & not observed & 3463 & 75 & $\mathrm{NH}_{2}$ sym. \\
\hline 5 N7H hydroxy-amino & & 3587 & 3582 & 80 & $\mathrm{OH}$ \\
\hline (7H enol syn) & & 3577 & 3570 & 40 & $\mathrm{NH}_{2}$ antisym. \\
\hline$A$ & & $3516^{3} ; 3515^{6}$ & 3520 & 83 & $\mathrm{~N} 7 \mathrm{H}$ \\
\hline origin : $32864 \mathrm{~cm}^{-1}$ & & 3462 & 3456 & 58 & $\mathrm{NH}_{2}$ sym. \\
\hline 7 N7H oxo-imino & & $3505^{3} ; 3503^{6}$ & 3509 & 104 & $\mathrm{~N} 7 \mathrm{H}$ \\
\hline$C$ & & $3497^{3} ;$ not observed in 6 & 3501 & 66 & $\mathrm{~N} 3 \mathrm{H}$ \\
\hline \multirow[t]{2}{*}{ origin : $33910 \mathrm{~cm}^{-1}$} & & $3450^{3} ; 3449^{6}$ & 3467 & 76 & $\mathrm{~N} 1 \mathrm{H}$ \\
\hline & & not observed & 3375 & 11 & NH imino \\
\hline 8 N7H oxo-imino & & $3506^{3} ; 3504^{6}$ & 3511 & 103 & $\mathrm{~N} 7 \mathrm{H}$ \\
\hline \multirow[t]{2}{*}{ origin : $33269 \mathrm{~cm}^{-1}$} & & 3456 & 3470 & 49 & $\mathrm{~N} 1 \mathrm{H}$ \\
\hline & & not observed & 3386 & 13 & $\mathrm{NH}$ imino \\
\hline
\end{tabular}

a: $\mathrm{NH}$ and $\mathrm{OH}$ stretches are scaled by specific factors ( 0.963 and 0.971 respectively).

occurrence of a tautomer-dependent fast relaxation process in the excited state. Additional experiments will be useful in order to support the present interpretation. In particular systematic gas phase scans either using two-color R2PI or fluorescence will be carried out to find spectral features of the "missing" tautomers. In this context, He droplets experiments on guanine and $9 \mathrm{MG}$ as well could also be welcome in order to determine the electronic absorption region of the 4 most stable forms.

The assignment of the R2PI spectra in terms of rare species is expected to be of broad significance since it may affect any flexible system whose excited state dynamics is strongly conformation-dependent. This should be expected in particular for complexes of guanine. ${ }^{23-27}$ As an example, the IR spectra of three conformers of guanine-water detected by $\mathrm{R}_{2} \mathrm{PI}^{26}$ exhibit common IR features with the minor tautomers of the guanine R2PI spectrum, suggesting that one observes hydrates of the rare tautomers. In the same spirit, guanine-containing dimers might be formed between one of the most stable forms and one of those actually detected by R2PI, which suggests enlarging the set of possible structures to account for observations. ${ }^{23,27}$

Finally, the difficulties encountered in recording the nearUV spectrum of the most stable tautomers of guanine, including the biologically relevant form, emphasize the importance of theoretical studies as a relevant alternative to experiment. Providing accurate electronic transition energies of the seven tautomers observed would greatly help spectroscopists to find the probably weak features of the "missing" tautomers. Alternatively, the presently revised experimental data pertaining to an enlarged set of tautomers should help understanding and refining the mechanism controlling the excited state relaxation dynamics in the DNA bases, ${ }^{2,11-13,17-20,22,28}$ in particular its dependence with the tautomeric form.

Acknowledgment. This work is dedicated to the memory of R.E. Miller, deceased in 2005.

Supporting Information Available: Table S1: Calculated harmonic NH/OH stretch frequencies of the 8 forms of Chart 1 . This material is available free of charge via the Internet at http://pubs.acs.org.

\section{References}

(1) Nir, E.; Grace, L.; Brauer, B.; de Vries, M. S. J. Am. Chem. Soc. 1999, 121,4896

(2) Nir, E.; Kleinermanns, K.; Grace, L.; de Vries, M. S. J. Phys. Chem. A 2001, 105, 5106

(3) Nir, E.; Janzen, C.; Imhof, P.; Kleinermanns, K.; de Vries, M. S. J. Chem. Phys. 2001, 115, 4604 .

(4) Nir, E.; Plutzer, C.; Kleinermanns, K.; de Vries, M. Eur. Phys. J. D 2002, 20, 317.

(5) Piuzzi, F.; Mons, M.; Dimicoli, I.; Tardivel, B.; Zhao, Q. Chem. Phys. 2001, 270, 205.

(6) Mons, M.; Dimicoli, I.; Piuzzi, F.; Tardivel, B.; Elhanine, M. J. Phys. Chem. A 2002, 106, 5088 .

(7) Chin, W.; Mons, M.; Dimicoli, I.; Piuzzi, F.; Tardivel, B.; Elhanine, M. Eur. Phys. J. D 2002, 20, 347.

(8) Chin, W.; Mons, M.; Piuzzi, F.; Tardivel, B.; Dimicoli, I.; Gorb, L.; Leszczynski, J. J. Phys. Chem. A 2004, 108, 8237.

(9) Ha, T. K.; Keller, H.-J.; Gunde, R.; Gunthard, H.-H. J. Phys. Chem. A 1999, 103,6612 .

(10) Hanus, M.; Ryjacek, F.; Kabelac, M.; Kubar, T.; Bogdan, T. V.;

Trygubenko, S. A.; Hobza, P. J. Am. Chem. Soc. 2003, 125, 7678.

(11) Gorb, L.; Kaczmarek, A.; Gorb, A.; Sadlej, A. J.; Leszczynski, J. J. Phys. Chem. B 2005, 109, 13770 .

(12) Langer, H.; Doltsinis, N. L. Phys. Chem. Chem. Phys. 2004, 6, 2742.

(13) Cerný, J.; Špirko, V.; Mons, M.; Hobza, P.; Nachtigallová, D. Phys. Chem. Chem. Phys. 2006, accepted; doi: 10.1039/b601629d.

(14) Choi, M. Y.; Miller, R. E. J. Am. Chem. Soc. 2006, 128, 7320

(15) Dong, F.; Miller, R. E. Science 2002, 298, 1227.

(16) Plützer, C.; Nir, E.; de Vries, M. S.; Kleinermanns, K. Phys. Chem. Chem. Phys. 2001, 3, 5466

(17) Chen, H.; Li, S. H. J. Chem. Phys. 2006, 124, 154315

(18) Shukla, M. K.; Leszczynski, J. J. Phys. Chem. A 2005, 109, 7775.

(19) Langer, H.; Doltsinis, N. L.; Marx, D. ChemPhysChem 2005, 6, 1734

(20) Sobolewski, A. L.; Domcke, W.; Hattig, C. Proc. Natl. Acad. Sci. U. S. A. 2005, 102, 17903.

(21) Mennucci, B.; Toniolo, A.; Tomasi, J. J. Phys. Chem. A 2001, 105 7126.

(22) Canuel, C.; Mons, M.; Piuzzi, F.; Tardivel, B.; Dimicoli, I.; Elhanine, M. J. Chem. Phys. 2005, 122, 074316.

(23) Nir, E.; Janzen, C.; Imhof, P.; Kleinermanns, K.; de Vries, M. S. Phys. Chem. Chem. Phys. 2002, 4, 732.

(24) Nir, E.; Janzen, C.; Imhof, P.; Kleinermanns, K.; de Vries, M. S. Phys. Chem. Chem. Phys. 2002, 4, 740

(25) Abo-Riziq, A.; Grace, L.; Nir, E.; Kabelac, M.; Hobza, P.; de Vries, M. S. Proc. Natl. Acad. Sci. U. S. A. 2005, 102, 20.

(26) Crews, B.; Abo-Riziq, A.; Grace, L.; Callahan, M.; Kabelac, M.; Hobza, P.; de Vries, M. S. Phys. Chem. Chem. Phys. 2005, 7, 3015.

(27) Bakker, J. M.; Compagnon, I.; Meijer, G.; von Helden, G.; Kabelac, M.; Hobza, P.; de Vries, M. S. Phys. Chem. Chem. Phys. 2004, 6, 2810.

(28) Fulscher, M. P.; SerranoAndres, L.; Roos, B. O. J. Am. Chem. Soc. 1997, 119,616 